\title{
Exposición laboral a fluidos corporales de riesgo en el Hospital Clínico Félix Bulnes Cerda durante 11 años de estudio
}

\author{
Julia Villarroel, M. Cecilia Bustamante, Iván Manríquez, M. Paz Bertoglia, María Mora y Natalie Galarce
}

\section{Occupational exposure risk to body fluids in the Felix Bulnes Hospital during eleven years}

Accidents with risk of occupational exposure to body fluids constitute more of a third of labor accidents. Objective: To describe the annual incidence of accidents with exposure to body fluids in the Felix Bulnes Hospital from1998 to 2008. Material and Methods: A retrospective analysis of reports from the Infection Control Committee. Results: During 11 years, there were 415 accidents with exposure to body fluids, with the cumulative incidence of 3,4\%(range $1.3 \%$ to $6 \%$ ). Sharp instrument accidents accounted for $92,5 \%$ of cases. The main health care providers affected were the paramedical technicians and the students. The highest frequency of accidents occurred in the obstetrical operating rooms $(20 \%)$ and in the central operating rooms $(17 \%)$. There were no cases of seroconversion and no exposure to HCV or HBV. The estimated costs were USD \$35638,6 or USD \$271 per 1000 staff per year. Conclusions: The incidence increased during the first years of the study and then remained stable since 2001, despite efforts in training personnel. Many factors contribute to the development of these accidents, such as lack of experience, type of clinical benefit and even daytime working hours.

Key words: Body fluids, occupational exposure, incidence.

Palabras clave: Fluidos corporales, accidente laboral, incidencia.

\section{Introducción}

1 1 personal de salud está enfrentado a diversos riesgos por exposición a sustancias biológicas, químicas y a accidentes con material corto punzante, los que constituyen una emergencia en infectología. Prevenir la exposición es la mejor forma de evitar la transmisión, y por cierto, la más económica.

Desde la aparición de la epidemia de SIDA, existe mayor conciencia sobre los riesgos de exposición en el ambiente laboral a virus de hepatitis B (VHB), virus de hepatitis $\mathrm{C}$ (VHC) y virus de inmunodeficiencia humana $(\mathrm{VIH})^{1}$.

Un tercio de los accidentes laborales notificados que afectan a funcionarios de la salud lo constituyen los accidentes corto-punzantes, con el consiguiente riesgo de infección por VHB, VHC, VIH y otros patógenos transmitidos por la sangre, riesgo relativo cuantificado para cada tipo de exposición por diversos estudios ${ }^{2,3}$. En Estados Unidos de América (E.U.A.), 4 millones de trabajadores de la salud presentan accidentes de trabajo por exposición a sangre y/o fluidos corporales, de los cuales 800.000 son secundarios a accidentes corto-punzantes ${ }^{3}$.

Hepatitis $B$ y hepatitis $C$. Históricamente la hepatitis $\mathrm{B}$ ha sido el patógeno más importante relacionado con la exposición ocupacional, siendo este riesgo diez veces mayor en trabajadores de la salud que en la población general ${ }^{4}$ Por otra parte, los trabajadores que llegan a contraer la hepatitis B tienen riesgo de desarrollar enfermedad hepática crónica ${ }^{5}$.

La posibilidad de infección por VHB es un riesgo que está relacionado con el tiempo de contacto con la sangre, así como la presencia de antígeno "e" en la fuente. Diferentes estudios efectuados en trabajadores de la salud, quienes sufrieron accidentes percutáneos con sangre portadora del VHB, han permitido determinar el riesgo de desarrollar hepatitis clínica si la sangre de la persona fuente era positiva tanto para el antígeno de superficie "s" (HBsAg) como para el antígeno "e" (HBeAg), con un riesgo de 22 a $31 \%$ y el de desarrollar evidencia serológica de infección fue de $37 \%$ a $62 \%$. La situación es diferente si la exposición es con sangre contaminada con HBsAg, pero con HBeAg negativo. En estos casos el riesgo de desarrollar hepatitis clínica fluctúa entre 1 y $6 \%$, y de desarrollar evidencia serológica de infección entre 23 y $37 \%$.

La sangre contiene los títulos más altos de VHB por sobre los otros fluidos corporales. Algunos fluidos como el líquido cefalorraquídeo, la bilis, lavado nasofaríngeo, saliva, leche materna, semen, sudor y líquido sinovial o desechos como las heces, contienen HBsAg (antígeno de superficie); sin embargo, la concentración de este antígeno es variable, 100 a 1.000 veces inferior que las partículas
Hospital Félix Bulnes Cerda, Santiago, Chile.

Servicio de Pediatría (JV, MCB). Médico becario de Pediatría (IM). Comité de Infecciones asociadas a la atención de salud (MM, NG). Magíster en Bioestadística (C) Universidad de Chile (MPB).

Los autores declaran no tener conflictos de interés hospital Fuentes de financiamiento: propias Recibido: 7 de diciembre de 2010 Aceptado: 6 de marzo de 2012

Correspondencia a: Julia Villarroel B. julia.villarroel@vtr.net 
infectantes de VHB; en consecuencia, la mayoría de los fluidos corporales no son vehículos eficientes de transmisión de VHB a pesar de la presencia en ellos del $\mathrm{HBsAg}^{7}$.

En relación al $\mathrm{VHC}$, éste no es transmitido tan eficientemente a través de la exposición ocupacional a sangre como la hepatitis $\mathrm{B}$. La incidencia media de seroconversión para el VHC después de una exposición percutánea ocupacional a una fuente positiva es de 1,8\% (rango 0-7\%). En todos los casos descritos la transmisión ocurrió a través de agujas con lumen ${ }^{8,9}$.

Existen otros agentes que ocupan las mismas formas de transmisión que los anteriormente mencionados, dentro de los cuales han ido cobrando relevancia el virus de la hepatitis $\mathrm{G}^{10}$ (virus ARN de la familia de los flavivirus al igual que el VHC), y el virus de la hepatitis D, el cual requiere co-infección con el VHB para llevar a cabo su ciclo completo de replicación.

Virus de inmunodeficiencia humana. El CDC de Atlanta, E.U.A., ha reportado al menos 57 casos comprobados y 138 casos probables de infección por VIH post exposición a fluidos corporales de alto riesgo ${ }^{3}$. En Europa se han confirmado 34 casos y hay 70 más con probable transmisión ocupacional en trabajadores de la salud.

En nuestro país se realizó un estudio en el Hospital Exequiel González Cortés que reportó la notificación de 130 exposiciones laborales durante los años 1992 a $1997^{11}$. Recientemente un estudio del Hospital Clínico de la Universidad de Chile, efectuado entre estudiantes de carreras de la salud, reportó 155 accidentes en los estudiantes durante los años 2003 a $2007^{12}$. Ninguno de los dos trabajos anteriores reportó seroconversión en el seguimiento.

\begin{tabular}{rrrr}
$\begin{array}{r}\text { Tabla 1. Tendencia de los accidentes de exposición a fluidos corporales durante el } \\
\text { período estudiado. Hospital Clínico Félix Bulnes Cerda años 1998 a 2008 }\end{array}$ \\
\hline Años & $\begin{array}{c}\text { Funcionarios y alumnos } \\
\text { Accidentes de exposición } \\
\text { a fluidos corporales }\end{array}$ & $\begin{array}{c}\text { Incidencia por 100 años/ } \\
\text { persona }\end{array}$ \\
\hline 1998 & 1.000 & 13 & 1,3 \\
\hline 1999 & 979 & 37 & 3,8 \\
\hline 2000 & 1.014 & 61 & 6 \\
\hline 01 & 1.041 & 42 & 4 \\
\hline 02 & 1.062 & 39 & 3,6 \\
\hline 03 & 1.077 & 32 & 2,9 \\
\hline 04 & 1.086 & 39 & 3,5 \\
\hline 05 & 1.113 & 44 & 3,9 \\
\hline 06 & 1.149 & 37 & 3,2 \\
\hline 07 & 1.197 & 34 & 2,8 \\
\hline 08 & 1.237 & 37 & 2,9 \\
\hline Total & 11.955 & 415 & 3,4 \\
\hline
\end{tabular}

Estudios prospectivos en trabajadores de la salud han estimado el riesgo de transmisión del VIH después de una exposición percutánea en $0,3 \%$ y en $0,09 \%$ después de una exposición de membranas mucosas ${ }^{13}$. A pesar que se han documentado episodios de transmisión del VIH ante la exposición de piel no intacta, el riesgo promedio de transmisión por esta vía no ha sido cuantificado con precisión, pero se estima que es menor al riesgo por exposición de membrana mucosa ${ }^{14-16}$.

Nuestro propósito es describir los accidentes por exposición laboral a fluidos corporales de riesgo y su tendencia en el tiempo en nuestro hospital, conocer sus causas, costos económicos involucrados, estamentos y servicios clínicos y/o de apoyo más afectados y, por último, describir la situación de vacunación del personal contra VHB.

\section{Material y Método}

Se efectuó un estudio descriptivo retrospectivo sobre el universo de registros de exposición laboral a fluidos de riesgo notificados en el Hospital Félix Bulnes Cerda (HFBC) entre los años 1998 y 2008, los que fueron proporcionados por el Comité de Infecciones Asociadas a la Atención de Salud (IAAS) de dicho centro hospitalario.

Se definió como personal de salud expuesto a experimentar accidentes con sangre y/o fluidos corporales de riesgo, a las personas cuya actividad incluye el contacto con sangre $u$ otros fluidos corporales que provengan de los usuarios en el ámbito de la salud. Se incluyeron a los funcionarios de los servicios clínicos y de apoyo del HFBC, además de los alumnos de pre y post-grado de las diferentes carreras de la salud de: Universidad de Santiago (estudiantes de medicina), Universidad Mayor (estudiantes de medicina, enfermería, obstetricia y kinesiología) e Instituto Santo Tomás (estudiantes de la carrera de técnicos en enfermería nivel superior). Los registros del número absoluto de personas que fueron catalogadas como personal de salud expuesto fueron otorgados por la oficina de recursos humanos y de registros de estudiantes en práctica del HFBC (Tabla 1).

Se definió el riesgo de la exposición según la normativa MINSAL $^{37}$ (Tabla 2).

Según la "Norma de manejo pos-exposición laboral a sangre en el contexto de la prevención de la infección por VIH" del MINSAL ${ }^{2}$, se tomaron exámenes para medir anticuerpos anti VIH a la persona fuente y a la persona expuesta. Además, como normativa interna del HFBC, se midió HBsAg y anticuerpos anti hepatitis C. Desde el año 2006, se contó con test rápido para VIH de tercera generación (BIO-RAD GENIEII HIV-1/HIV-2. Laboratorio Galénica) el que se efectúo en la Unidad de Medicina Transfusional del mismo hospital, lo cual permitió obtener los resultados de las muestras en el lapso 
de una hora, minimizando la angustia del afectado y el uso de terapia anti-retroviral profiláctica.

Se indicó profilaxis con zidovudina (AZT) + lamivudina (3TC) (Combivir ${ }^{\circledR}$ GSK), dos cápsulas al día por 48 horas (se entregaron 4 cápsulas), a todas las personas que sufrieron exposición de riesgo con fuente conocida, mientras se esperaba los resultados de los análisis de sangre solicitados, de acuerdo a la norma MINSAL ${ }^{2}$. Cuando el test de ELISA para VIH resultó negativo para la persona fuente, se suspendía inmediatamente la profilaxis indicada. Cuando el resultado fue positivo, las personas expuestas recibieron el resto de profilaxis para completar 30 días con Combivir ${ }^{\circledR}$ y de inmediato fueron derivadas al Hospital San Juan de Dios de Santiago de Chile, centro de referencia en infectología de adultos. Se realizó seguimiento con nuevos exámenes de control (test de ELISA para VIH, HBsAg y anticuerpo anti-hepatitis C), a los 3 y 6 meses post exposición a las personas que experimentaron exposición de riesgo con una fuente conocida seropositiva para VIH, según la norma MINSAL ${ }^{2}$. De igual manera, se realizó seguimiento con exámenes de control a las personas que experimentaron exposición de riesgo con una fuente conocida seronegativa para $\mathrm{VIH}$, de acuerdo a normativa interna del Comité IAAS del HFBC.

Finalmente, se administró vacuna anti-hepatitis B a todos los expuestos que no contaban con esquema de vacunación o si éste estaba incompleto. No se contempló la medición de títulos de anticuerpos anti hepatitis B para determinar títulos protectores en los vacunados expuestos a VHB.

A pesar que el Comité de IAAS del HFBC había contemplado el uso de inmunoglobulina específica antihepatitis $\mathrm{B}$ en los posibles casos de exposición de riesgo a fluidos contaminados con dicho virus, no fue necesario su uso, pues no existieron personas-fuente con examen positivo para HBsAg.

Costos involucrados: Se analizaron los costos de la consejería y toma de muestra (valor hora del trabajo de enfermera(o), valor del test de ELISA para VIH, vacuna anti hepatitis $\mathrm{B}$, Combivir ${ }^{\circledR}$, determinación de $\mathrm{HBsAg}$, y los anticuerpos para hepatitis $\mathrm{C}$, considerando el valor de
FONASA nivel 1 (valor año 2009). También se considera el costo estimado referente a reposo laboral de los funcionarios afectados que recibieron tal beneficio.

Se definieron tres tipos de situaciones para calcular los costos

- Situación 1: accidentes con fuente conocida, sin riesgo de exposición (209 personas expuestas).

Se consideró una toma de exámenes para la persona expuesta y para la persona fuente, incluyendo además una hora de trabajo de enfermera.

- Situación 2: accidentes con fuente desconocida, exposición sin riesgo (136 personas expuestas).

En este caso se consideró la extracción de una muestra de sangre de la persona afectada más la hora de trabajo de enfermera.

- Situación 3: accidentes con fuente conocida con riesgo de exposición (70 personas expuestas, 64 con fuente seronegativa para VIH y 6 con fuente seropositiva para $\mathrm{VIH})$.

Se consideró la toma de exámenes a la persona-fuente y a la persona expuesta inmediatamente después de producido el accidente. Posteriormente, se realizó seguimiento a todos los expuestos de esta situación, con exámenes de control a los 3 y 6 meses post exposición. Se suma a los exámenes el costo de Combivir ${ }^{\circledR}$ por 30 días de profilaxis a las 6 personas que tuvieron exposición a VIH confirmada por test de ELISA, la hora de trabajo de enfermera y el costo estimado de reposo laboral por 30 días que cada uno recibió.

A los 64 expuestos a sangre y/o fluidos de personafuente seronegativa para VIH, sólo se consideró el costo de cuatro comprimidos de Combivir ${ }^{\circledR}$, indicados como profilaxis inicial en espera de confirmación por el test de ELISA.

Finalmente, se realizó análisis de costos al total de dosis de vacuna anti-hepatitis B que debió administrarse a las personas expuestas que no tenían completo su esquema de vacunación o que simplemente no habían recibido dosis alguna. En estos casos se consideró sólo el costo de completar el esquema de vacunación y la hora de trabajo de enfermera.

\section{Tabla 2. Riesgo de exposición según normativa MINSAL}

Exposición con riesgo Exposición sin riesgo

Herida profunda que causa sangrado provocado por un instrumento con lumen lleno de sangre o fluido corporal de riesgo o a simple vista contaminado con sangre o fluido corporal de riesgo

Herida con instrumento que no está a simple vista contaminado con sangre o fluido corporal con riesgo

Exposición de mucosas o de heridas a sangre o fluidos corporales a Herida superficial que no causa sangrado

simple vista contaminados con sangre

Derrame de sangre o fluido corporal con riesgo, en una solución de

continuidad de la piel tales como heridas, dermatosis o eczema

Exposición de piel intacta o sana con sangre o fluido corporal de cualquier riesgo 


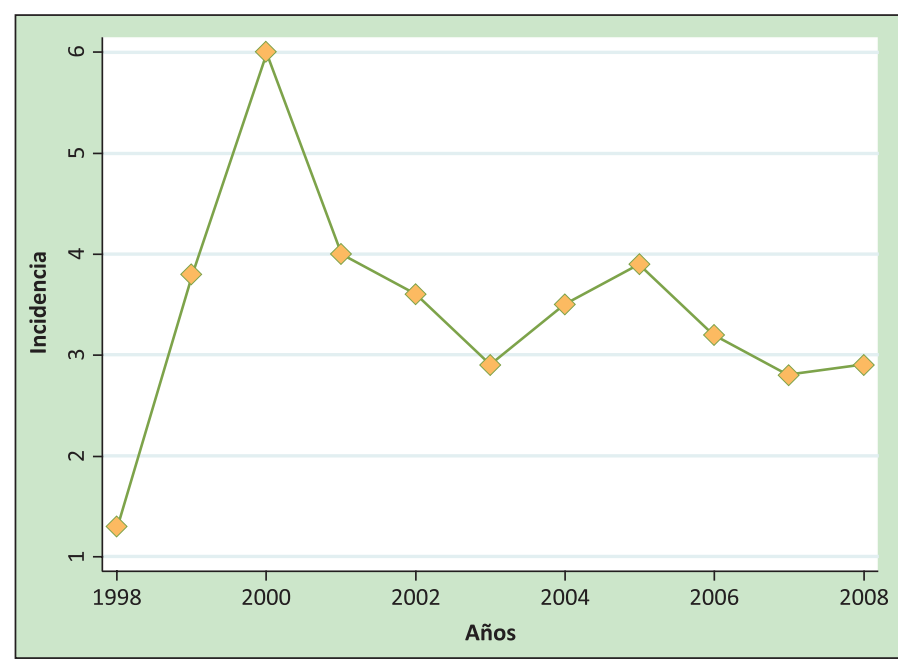

Figura 1. Incidencia de exposición accidental a fluidos corporales. Hospital Clínico Félix Bulnes Cerda años 1998 a 2008. Nota: La incidencia de accidentes de exposición a fluidos corporales está referida por 100 funcionarios/alumnos año, destacándose una tendencia estable.

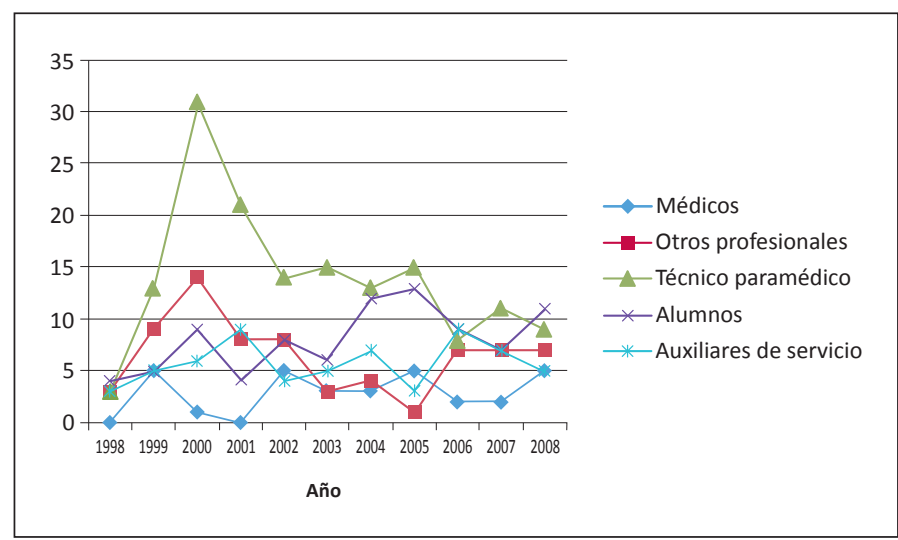

Figura 2. Frecuencia de exposiciones accidental a fluidos corporales por estamento durante el período en estudio. Hospital Clínico Félix Bulnes Cerda 1998 a 2008. Nota: El gráfico refleja la frecuencia de eventos por año, destacándose una tendencia a la disminución de exposiciones en el grupo de técnicos paramédicos.

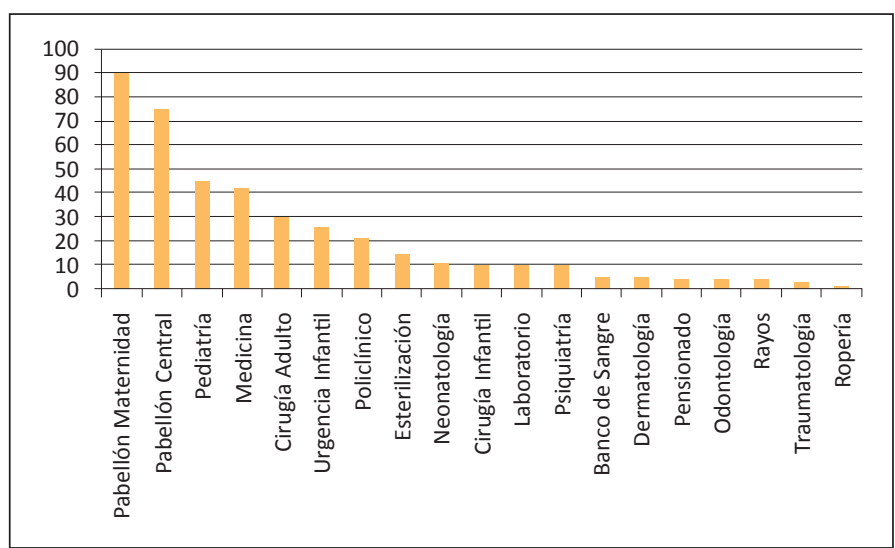

Figura 3. Distribución de frecuencia de exposiciones a fluidos corporales según Servicio Clínico Hospital Clínico Félix Bulnes Cerda 1998 a 2008.

\section{Resultados}

Tendencia de los accidentes. Se registró un total de 415 accidentes notificados por exposición a sangre y/o fluidos corporales de riesgo durante los 11 años de seguimiento. En el año 1998 se notificaron sólo 13 accidentes laborales de esta naturaleza, lo cual podría corresponder a una sub-notificación. La incidencia en los distintos años de seguimiento se muestra en la Figura 1.

Tipos de exposición. El tipo de exposición más frecuente fue el percutáneo con 384 casos $(92,5 \%)$. Las mucosas se vieron afectadas en $7 \%$ (29) y sólo en $0,1 \%$ la piel no intacta ( 2 casos).

Estamentos afectados. Se pudo determinar que los estamentos más afectados fueron los técnicos paramédicos con 148 personas expuestas (36\%) y los alumnos de pre y post grado con 87 personas expuestas (21\%). Los auxiliares de servicio registraron 58 personas expuestas (14\%) (Figura 2).

Exposición por servicio clínico. El servicio en el cual se produjo el mayor número de exposiciones laborales a sangre y/o fluidos humanos fue pabellones quirúrgicos de maternidad con 84 personas expuestas $(20 \%)$, seguido de pabellones quirúrgicos centrales con 72 personas expuestas (17\%) (Figura 3).

Del total de exposiciones notificadas durante el período estudiado, 295 (71\%), ocurrieron en horario diurno.

Condición de la persona-fuente. La persona-fuente fue conocida en 256 accidentes (62\%). La exposición a sangre $y / 0$ fluidos corporales de una persona fuente infectada por VIH ocurrió en 6 casos, no registrándose fuentes portadoras de hepatitis B o C. No hemos observado seroconversión en este período.

Causal del accidente. Cabe destacar que del total de eventos registrados, 268 correspondían a situaciones prevenibles $(64,4 \%)$ y los 147 restantes a eventos fortuitos $(35,4 \%)$, en los cuales no se produjo transgresión alguna a las normas.

Del total de eventos prevenibles, 159 casos $(38,3 \%)$, correspondieron a accidentes por mala eliminación de las agujas en las cajas de bio-seguridad. En 93 casos $(22,4 \%)$, no se usaron las medidas protectoras adecuadas, mientras que en 16 casos (3,9\%), el accidente se produjo al re-capsular las agujas.

Hasta el año 2002 se produjeron lesiones por recapsular agujas, práctica errada que ha ido desapareciendo gracias a la capacitación continua del personal de salud. Sin embargo, la eliminación de material corto-punzante en recipientes distintos a las cajas de bio-seguridad y el no 
uso de las medidas protectoras estándares, son prácticas que se mantienen, a pesar de la capacitación y de contar con los insumos necesarios.

Profilaxis contra hepatitis $B$. Referente al estado de vacunación anti-hepatitis B del personal, se pudo observar que en los dos primeros años de estudio, $98 \%$ de las personas expuestas no se encontraban vacunadas, situación que se revirtió hasta lograr en el año 2008 una cobertura de $62 \%$ entre los funcionarios. Esto fue posible gracias a programas de capacitación y de vacunación realizados.

Durante el período estudiado no fue necesario el uso de inmunoglobulina anti-hepatitis $\mathrm{B}$, pues no se registraron personas fuente portadoras de HBsAg.

Seroconversión. En seis oportunidades la personafuente arrojó resultado positivo en el test de ELISA para VIH y como las exposiciones habían sido de riesgo en los seis casos, se indicó profilaxis con Combivir ${ }^{\circledR}$ por 30 días a las personas expuestas, de acuerdo a la norma MINSAL $^{6}$, quienes inmediatamente eran derivados al centro de referencia, pues durante los años de seguimiento el HFBC no contaba con médico(a) especialista en Infectología de adultos.

Finalmente, se realizó consulta telefónica a las seis personas expuestas de riesgo ya descritas, a fin de conocer su adhesión al tratamiento y protocolo de seguimiento, posibles efectos secundarios o reacciones adversas a la farmacoterapia, existencia de seroconversión, tiempo de reposo laboral indicado y posibles alteraciones psicosociales secundarias a la exposición.

Las seis personas contactadas consintieron en informar sobre las interrogantes antes expuestas, destacando 100\% de adhesión al tratamiento farmacológico y al protocolo de seguimiento. Cuatro personas refirieron haber padecido molestias gastrointestinales leves, (náuseas, vómitos y dolor abdominal), durante el mes de profilaxis con la terapia de Combivir $\AA$, no requiriendo atención médica de urgencia ni hospitalización por esa causa. Las dos personas restantes no refirieron síntomas durante la profilaxis. El ausentismo laboral fue de 30 días en los seis casos en cuestión. En ningún expuesto hubo seroconversión para VIH o alguno de los patógenos estudiados.

Por último, es necesario destacar que todos los sujetos expuestos refirieron alteración cualitativa de su calidad de vida durante el período de seguimiento, manifestando síntomas como angustia, ansiedad e irritabilidad, afectando también a sus respectivas familias, sus vidas de pareja y actividad sexual.

Costos observados. En relación a los costos económicos observados en las tres situaciones descritas se concluye: (Valor del dólar, tipo de cambio actualizado al día 10 de noviembre de 2011).
Situación 1 (accidentados con fuente conocida, exposición sin riesgo). Se notificaron 209 personas expuestas con un costo observado total de $\$ 5.488 .340$ lo que corresponde a \$ 41.734 pesos cada 1.000 funcionario/ alumno por año (USD \$10,972 o USD \$ 83,43 por 1.000 funcionario/alumno por año).

Situación 2 (accidentados con fuente desconocida, exposición sin riesgo). Se notificaron 136 personas expuestas con un costo observado total de $\$ 1.852 .320$ lo que corresponde a $\$ 14.085$ pesos por 1.000 funcionario/ alumno por año (USD \$3,703 o USD \$28,16 por 1.000 funcionario/alumno por año).

Situación 3 (accidentados con fuente conocida, exposición con riesgo). Se notificaron 70 personas expuestas con un costo observado total de \$5.049.092 lo que corresponde a $\$ 38.394$ pesos por cada 1.000 funcionarios/ alumnos por año (USD \$ 10,094 o USD \$ 76,7 dólares por cada 1.000 funcionario/alumno por año).

Además de las situaciones antes planteadas se agrega a este análisis de costos los ítems referentes a vacunación anti hepatitis B. De las 415 personas expuestas se identificaron 101 personas con esquema de vacunación anti-hepatitis B incompleto o inexistente. El costo total de vacunación para estas personas fue de $\$ 3.330 .000$ (USD $\$ 6,657)$, lo que corresponde a $\$ 25.322$ pesos por cada 1.000 funcionario/alumno por año (USD \$ 50,6 dólares por 1.000 funcionario/alumno por año). Se desconoce el nombre comercial de las vacunas anti-hepatitis B utilizadas, por razones económicas fue frecuente el cambio de proveedor. El organismo encargado de proveer las dosis de vacunas al HFBC es el Servicio de Salud Occidente.

Por último, se consideró agregar a este análisis el costo de los días de reposo laboral secundarios a los accidentes por exposición laboral a fluidos de riesgo. En este estudio se registraron seis funcionarios, todos técnicos paramédicos, que recibieron indicación médica de reposo laboral, por 30 días cada uno. El costo estimado por reposo laboral de 30 días para un funcionario perteneciente al estamento de técnicos paramédicos es de \$262.194, valor entregado por la oficina de recursos humanos del establecimiento. En suma, los costos estimados por concepto reposo laboral alcanzaron el valor de \$ 1.573.164 (USD \$ 3,145). Lo que corresponde a $\$ 11.962$ pesos por cada 1.000 funcionario/ alumno por año (USD $\$ 24$ dólares por 1.000 funcionario/ alumno por año.

El total de costos estimados secundarios a exposición laboral a fluidos de riesgo durante los 11 años estudiados alcanzó un valor de $\$ 17.292 .916$. (USD \$34,571) lo que corresponde a $\$ 131.500$ pesos por 1.000 funcionario/ alumno por año (USD \$263 dólares por 1.000 funcionario/ alumno por año). 


\section{Discusión}

En el presente estudio, 92,5\% de los accidentes laborales por exposición a sangre y/o fluidos corporales fueron por vía percutánea (384 casos), valor elevado al comparar con lo descrito en la literatura médica, donde se ha observado que las agujas con lumen causan $85 \%$ del total de accidentes ${ }^{17,18}$. Asimismo, 1,44\% de las exposiciones laborales a sangre y fluidos corporales registradas en este estudio (seis personas expuestas), provenían de persona fuente seropositivas para VIH, lo cual concuerda con los valores registrados en la literatura médica nacional ${ }^{19,20}$.

Se observó que los técnicos paramédicos y los alumnos de pre y post grado, fueron los más frecuentemente afectados, a diferencia de estudios extranjeros que indican a las enfermeras como el estamento más afectado ${ }^{21-23}$. Con respecto a los alumnos, podemos inferir que la escasa experiencia en la práctica clínica, el poco desarrollo de habilidades manuales, la existencia de brechas de conocimiento en temática sanitaria y la escasa conciencia que en general tienen sobre los riesgos de infecciones intrahospitalarias, representan en sí una conducta de riesgo para la aparición de accidentes biológicos como los estudiados ${ }^{24,25}$. Algunos estudios efectuados en E.U.A., informaron de al menos una exposición a sangre durante los dos años de práctica clínica previa al período de pregrado $^{26,27}$. Estudios realizados en Colombia, informaron que $31,4 \%$ de los estudiantes e internos de medicina tienen al menos un accidente por cada seis meses de actividad académica $^{28}$, e incluso la incidencia puede ser mucho mayor, como lo señala un estudio realizado a estudiantes de post-grado de medicina durante su período de internado en algunos hospitales de Perú, entre quienes se informó que durante su año de práctica, hasta 95,6\% tuvieron al menos un accidente biológico ${ }^{29,30}$.

A pesar que las guías de precauciones estándares para prevenir infecciones ocupacionales entre los trabajadores de la salud enfatizan el uso apropiado de medidas de barrera y la disposición correcta de agujas y corto-punzantes ${ }^{31}$ en general, en contenedores especialmente diseñados para tales efectos, no siempre son seguidas a cabalidad, como fue posible apreciar en esta experiencia. Lo anterior también ha sido señalado por autores como Maclan y cols. ${ }^{32}$, quienes reportan en su estudio el uso de guantes sólo en $85 \%$ de todos los procedimientos, de protección ocular en $47 \%$, uso de bata $18 \%$, y mascarilla $4 \%$. Además, estos autores identificaron como principales motivos para no utilizar las precauciones estándares a factores como "falta de tiempo" en $61 \%$ de los encuestados y el hecho de que las barreras de protección son molestas o estorban al personal, en $29 \%$.

El riesgo de experimentar accidentes guarda relación con el nivel de conocimientos del personal de salud sobre el tema, tal como lo muestran Kim y cols. ${ }^{33}$, donde sólo
$20 \%$ del personal de urgencias logra identificar el riesgo que implica el no tener un esquema de vacunación completa contra hepatitis B; 49\% sabe que la eficacia de la vacunación está por sobre $90 \%$ y $43 \%$ sabe de la importancia de administrar una terapia anti-retroviral pocas horas después del accidente; $87 \%$ tenían completo el esquema de vacunación contra hepatitis B y $49 \%$ conocían la alta eficacia de la vacunación.

Los servicios de salud cuentan con programas de prevención y control de estos accidentes; no obstante, estos programas no han logrado el resultado esperado en cuanto a la sensibilidad de dicho sistema, ya que la literatura médica extranjera describe cifras de sub-notificación que varían entre 20 y $80 \%{ }^{34,35}$. Al evaluar el porcentaje de accidentes corto-punzantes con exposición a sangre que no fueron notificados, diversos autores encontraron tasas de $\sim 40 \%$ y en estudios realizados por el CDC en hospitales norteamericanos, el índice de sub-notificación varió de 45 a $80 \%$, dependiendo del tipo de institución y de la categoría profesional, correspondiendo al estamento médico los mayores porcentajes de sub-notificación ${ }^{35}$. A nivel nacional no hay trabajos publicados que demuestren el grado de sub-notificación existente.

Los programas de prevención han permitido establecer estrategias, evaluar el impacto de las medidas de prevención ${ }^{36,37}$, así como reducir los riesgos y contener los costos, ya que los valores de los tratamientos de una enfermedad ocasionada por algunos de los agentes que se transmiten a través de una exposición percutánea o por contacto de mucosas supera con creces el costo de las medidas de prevención y de profilaxis ${ }^{38,39}$.

En el presente estudio los accidentes por exposición a sangre y/o fluidos corporales de riesgo mostraron tasas anuales que fluctuaron entre 1,3 y $6 \%$, tasas que, según lo expresado en la literatura científica, están muy por debajo de la realidad y que en parte se ven demostradas por el reducido número de accidentes notificados en 1998, situación que ha mejorado con programas de capacitación. No obstante, la incidencia se ha mantenido relativamente estable a partir del año 2001, a pesar de las capacitaciones efectuadas por el Comité de IAAS en los diferentes servicios y de contar con una adecuada dotación de cajas de bio-seguridad para depositar el material corto-punzante.

En los servicios quirúrgicos la frecuencia observada de lesiones fue superior a la demostrada en los otros servicios clínicos, al igual que lo descrito en la literatura científica $^{40}$, lo que puede tener relación con la intensidad de la exposición a materiales corto-punzantes.

La mayoría de las exposiciones estudiadas se produjeron de día, (295, que corresponde a $71 \%$ ), lo cual pudiera estar relacionado con la tensión y sobrecarga laboral que probablemente ocurre en la jornada laboral diurna, factores que podrían constituir un mayor riesgo para el desarrollo de estos accidentes laborales. 
Durante el período en estudio no observamos seroconversión para alguno de los agentes virales estudiados.

El fenómeno de los accidentes por exposición a sangre y fluidos corporales durante el período analizado demuestra en un primer momento una mejora de la notificación, manteniéndose relativamente estables en el resto del período, a pesar de haberse realizado capacitación y adquisición de medios de bio-seguridad.

Como se trata de un fenómeno localizado mayoritariamente en algunos servicios específicos, la capacitación debe enfocarse en ellos, abordando los problemas más frecuentes, y con carácter obligatorio para quienes ingresan a esos servicios. Esta capacitación debe incorporar a los docentes responsables de los alumnos en práctica, los cuales deben ser capacitados como parte de la inducción a la práctica.

De este estudio se puede concluir que si se aplicaran medidas de prevención de los accidentes con fluidos corporales de riesgo, se podría haber evitado 3,9\% de accidentes por recapsular agujas, 22,4\% por no utilizar medidas de prevención y $38,3 \%$ por eliminación inadecuada de material corto-punzante y/o desechos y fluidos orgánicos. Todo lo anterior habría ayudado a reducir considerablemente los costos de pesquisa, tratamiento profiláctico y seguimiento de las personas expuestas.

Las exposiciones de riesgo biológico en el personal de salud son frecuentes, no tienden a disminuir a pesar de programas de capacitación y originan un elevado costo.

Los autores reconocen es este estudio la existencia de un posible sesgo de información, en vista que los datos analizados se obtuvieron de los registros de notificación de accidentes laborales por exposición a sangre y fluidos corporales y es factible considerar la existencia de accidentes que no fueron notificados, ya fuese por decisión personal, por desinformación respecto a la normativa vigente o desconocimiento de la existencia de un profesional responsable del sistema de vigilancia y seguimiento de los accidentes del trabajo y enfermedades profesionales, que por norma ministerial ${ }^{6}$ debe existir en todo centro de salud chileno. No está demás relatar que incluso una de las personas expuestas y notificada en este estudio, de riesgo y con fuente conocida seropositiva para VIH, no informó a su jefatura directa y mucho menos a la persona encargada de vigilancia epidemiológica del establecimiento de su condición de expuesto, sino hasta 72 horas después de ocurrido el evento, ingresando tardíamente al flujograma de manejo post exposición. A pesar de ello, favorablemente no existió seroconversión en éste ni en otro caso durante el período de estudio.

Agradecimientos: A Anabella Arredondo, pediatra epidemióloga, quien efectuó una revisión de este trabajo.

\section{Resumen}

Los accidentes por exposición a fluidos corporales de riesgo constituyen más de un tercio de los accidentes laborales. Objetivo: Describir la incidencia anual de accidentes por exposición a fluidos corporales de riesgo en el Hospital Clínico Félix Bulnes Cerda durante los años 1998 a 2008. Material y Métodos: Estudio descriptivo, retrospectivo, de análisis de reportes del Comité de infecciones asociadas a la atención de salud (IAAS). Resultados: Se registraron 415 accidentes por exposición a fluidos corporales de riesgo, siendo la incidencia acumulada de 3,4\% (rango 1,3 a 6,0\%) durante los 11 años estudiados. Los accidentes corto-punzantes constituyeron $92,5 \%$ de los casos. Los técnicos paramédicos y alumnos de carreras de la salud fueron los estamentos más afectados. La mayor frecuencia de accidentes ocurrió en los pabellones quirúrgicos de maternidad (20\%) y en los pabellones centrales (17\%). No se registraron casos de seroconversión y no hubo exposiciones a VHC o VHB. Los costos estimados fueron $\$ 17.292 .916, \$ 131.500$ por 1.000 funcionario/ alumno por año (USD \$34,571 o USD \$ 263 por 1.000 funcionario/alumno por año). Conclusiones: La incidencia aumentó durante los primeros años del estudio para luego mantenerse estable desde el año 2001, a pesar de los esfuerzos realizados en capacitación. Muchos factores favorecen el desarrollo de estos accidentes, como la falta de experiencia, el tipo de prestación clínica e incluso la jornada laboral diurna.

\section{Referencias bibliográficas}

1.- Eatin J, Taylor K. The psychosocial impact of AIDS on health workers. AIDS 1990; 4 (supl 1): 257-62.

2.- Norma $\mathrm{N}^{\circ} 48$ de manejo post-exposición laboral a sangre en el contexto de la prevención de la infección por VIH. MINSAL Octubre 2000.

3.- Do A, Cielsielski C, Metler R, Hammett T, Li J, Fleming P. Occupationally acquired human immunodeficiency virus (HIV) infection: National case surveillance data during 20 years of the HIV epidemic in the United States. Infect Control Hosp Epidemiol 2003; 24 (2): 86-96.

4.- Cardo D M, Culver D H, Ciesielski C A, Srivastava P U, Marcus R, et al. A case-control study of HIV seroconversión in health care workers after percutaneaus exposure. N Engl J Med 1997; 337: 1485-90.

5.- Anonimous. Guidelines for the management of occupational exposures to HBV, HCV and HIV and recommendations for postexposure prophylaxis. MMWR Morb Mortal Wkly Rep 2001; 50 (RR-11): 1-52.
6.- Werner B, Grady G. Accidental hepatitis B surface antigen positive inoculations: use of $\mathrm{e}$ antigen to estimate infectivity. Ann Intern Med 1982; 97: 367-9.

7.- Shapiro C. Occupational risk of infection with hepatitis B and hepatitis C virus. Surg Clin North Am 1995; 75: 1047-56.

8.- Puro V, Petrosillo N, Ippolito G. Risk of hepatitis $\mathrm{C}$ seroconvertion after occupational exposure in health care workers. Am J Infect Control 1995; 23: 273-7.

9.- Lauphear B, Linnemann C, Cannon C, De 
Ronde M, Pendy L, Kerley L. Hepatitis C: virus infection in healtcare workers; risk of exposure and infection. Infect Control Hosp Epidemiol 1994; 15: 745-50.

10.- Ruiz V, Benítez N. Algunas consideraciones sobre la hepatitis G. Rev Cubana Med Gen Integ 1999; 15 (3): 328-33.

11.- Mendoza C, Barrientos C, Vásquez R, Panizza V. Exposición laboral a sangre y fluidos corporales. Experiencia en un hospital pediátrico. Rev Chilena Infectol 2001; 18 (1): 28-34.

12.- Fica A, Jemenao I, Ruiz G, Larrondo M, Hurtado C, Muñoz G, et al. Accidentes de riesgo biológico entre estudiantes de carreras de la salud. Cinco años de experiencia. Rev Chilena Infectol 2010; 27 (1): 34-9.

13.- Anonimous. Case-control study of HIV seroconversion in health-care workers after percutaneous exposure to HIV-infected bloodFrance, United Kingdom, and United States, January 1988-August 1994. MMWR Morb Mortal Wkly Rep 1995; 44 (50): 929-36.

14.- De Juanes J, García de Codes A, Arrazola M, González A. Occupational exposure to human immunodeficiency virus in hospital health care workers in Spain. Vaccines 2007; 8 (1): 4-8.

15.- Hippolito G, Carli G, Puro V. Device-specific risk of needle sticks injury in Italian health care workers. JAMA 1994; 272: 607-10.

16.- Gerbending J. Occupational exposure to HIV in health care setting. N Engl J Med 2003; 348 (9): 826-33.

17.- Hernández M, Camoins M, Martínez E, Ramos F, García de Codes I, Arribas J. Occupational exposure to blood and biological material in healthcare workers. Med Clin (Barc) 2004; 122: 81-6.

18.- Morales-Aguirre J. Frecuencia y mecanismos de exposición accidental a productos biológicos potencialmente infecciosos en el personal de la salud. Bol Med Hosp Infant Mex 2006; 63 (4): 247-54.

19.- Herrera R, Bavestrello L, Barthel E, Nercelles P, Peirano L, Villarroel M L. Resultados del programa de prevención de exposiciones laborales a cortopunzantes. Primer Congreso Panamericano de Infecciones Intrahospitalarias.
Viña del Mar. año 1995.

20.- Fuentes S, Almarz L. Accidentes cortopunzantes en funcionarios hospitalarios. Primer Congreso Panamericano de Infecciones Intrahospitalarias. Viña del Mar. año 1995.

21.- Kosgeroglu N, Ayrance U, Vandareli E, Dincer S. Occupational exposure to hepatitis infection among Turkish nurses: Frequency of needle exposure, sharps injuries and vaccination. Epidemiol Infect 2003; 132: 27-33.

22.- Clarke S, Schubert M, Korner T. Sharp-device injuries to hospital staff nurses in 4 countries. Infect Control Hosp Epidemiol 2007; 28 (4): 473-8.

23.- Bueno L, Álvarez M, Guanche H, García E. Prevalencia de lesiones por objetos cortopunzantes en el personal de enfermería de unidades de terapia quirúrgicas. Rev Cubana Hig Epidemiol 2007; 45 (2): 1561-3003.

24.- Koening S, Chu J. Medical student exposure to blood and infectious body fluids. Am J Infect Control 1995; 23: 40-3.

25.- Birenbaum D, Wohli A, Duda B, Runyon M, Stearns B, Willett M. Medical students' occupational exposures to potentially infectious agent. Acad Med 2002; 77: 185-9.

26.- Gamester C, Tilzey A, Banatvala J. Medical students' risk of infection with bloodborne viruses at home and broad: questionnaire survey. Br Med J 1999; 318: 158-60.

27.- Shen C, Jagger J, Pearson R D. Risk of needle stick and sharp object injuries among medical students. Am. J Infect Control 1999; 27: 435-7.

28.- Herrera A, Gómez R. Accidentes por riesgo biológico en los estudiantes de medicina y médicos internos de la Universidad Tecnológica de Pereira. Resúmenes, XVII Congreso Científico Internacional, FELSOCEM, Lima Perú 2002: 60.

29.- Díaz L, Cadena L. Riesgo de infección por hepatitis B entre estudiantes de medicina peruanos luego de exposición a sangre y líquidos corporales. Rev Gastroenterol Perú 2003; 23 (2): 107-10.

30.- Ramos S, Castillo C, Reyes N, Villalba V. Accidentes laborales con exposición a fluidos corporales en internos de medicina de Lima metropolitana. CIMEL Revista Científica 2001;
6: 26-30.

31.- Beekmann S, Vaughn T, McCoy K, Ferguson K, Torner J, Woolson R, et al. Hospital bloodborne pathogens programs: Program characteristics and blood and body fluid exposure rates. Infect Control Hosp. Epidemiol 2001; 22 (2): 73-82.

32.- Maclan A, Raafat A, Hunt J. Barrier precautions in trauma: is knowledge enough? J Trauma 2002; 52: 540-3.

33.- Kim L, Evanoff B, Parks R. Compliance with universal precautions among emergency department personnel: Implications for prevention programs. Am J Infect Control 1999; 27: 453-5.

34.- Magnione C, Geberding J, Cummings S. Occupational exposure to HIV: frecuency and rates of underreporting of percutaneous and mucocutaneous exposures by medical house staff. Am J Med 1991; 90: 85-91.

35.- Rabaud C, Zanea A, Mur J, Blench M, Dazy D, May T, et al. Occupational exposure to blood: Search for a relation between personality and behavior. Infect Control Hosp. Epidemiol 2000; 21 (9): 564-74.

36.- Nercelles P, Herrera R, Guerra S. Resultados de un programa de prevención de exposiciones laborales a material cortopunzante. Libro de resúmenes del Primer Congreso Panamericano de IIH 1995.

37.- Comisión Nacional de SIDA. Normas de manejo post-exposición laboral a sangre en el contexto de la prevención de la infección por VIH. Octubre 2000

38.- Rosenthal V. Costo de los accidentes con sangre y fluidos corporales en el personal de salud. IX Congreso Chileno de IIH y Epidemiología Hospitalaria 4-7 Diciembre 2000.

39.- Kallenborn J, Price T, Carricor, Davison A. Emergency department management of occupational exposure: Cost analysis of rapid HIV test. Infect Control Hosp Epidemiol 2001; 22: 289-93.

40.- García de Codes A, Juanes J, Arrazola M, Jaén F, Sanz M, Lago E. Accidentes con exposición a material biológico contaminado por VIH en un hospital de tercer nivel de Madrid (1986-2001). Rev Esp Salud Pública 2004; 78: 1-10. 